\title{
MARUINS (CERATOPOGONIDAE: DIPTERA) DO ESTUÁRIO DO RIO PARÁ E DO LITORAL DO ESTADO DO PARÁ, BRASIL
}

\section{Rosimeire Lopes da Trindade \& Inocêncio de Sousa Gorayeb}

\begin{abstract}
Biting Midges (Ceratopogonidae: Diptera) of the Para River Estuary and Litoral of Para State, Brazil - The present study surveyed the species of hematophagus biting midges in coastal areas of Para State, Brazil. Two collecting sites were chosen, one a beach on the coast (Praia de Crispim, near Marapanim) and another in the Para River estuary (Outeiro Island, near Belem). Collections were made monthly in 2003, from April to October in Outeiro and the months of June, July, September, and October in Marapanim. Human bait and CDC traps were used. In total 4003 specimens were collected: 18 species of Culicoides Latreille and one of Leptoconops Skuse. L. brasiliensis (Lutz) was the only species common to both sites. The two most abundant species in Outeiro were $C$. batesi Wirth \& Blanton and C. denisae Clastrier, while in Marapanim these were C. maruim Lutz and C. phlebotomus (Williston).
\end{abstract}

Key words: Insecta, Diptera, Ceratopogonidae, Brazil, Amazon River estuary, coastal region

Coordenação de Zoologia, Entomologia, Museu Paraense Emílio Goeldi (MCT), Avenida Perimetral 1901, Bairro Terra-firme, CEP: 66017-970 Belém, Pará, Brasil. rltrindade@museu-goeldi.br

Recebido em: 05/11/2004.

Aceito em: 14/03/2005. 


\section{Resumo}

O presente trabalho teve como objetivo fazer o levantamento das espécies de ceratopogonídeos hematófagos (maruins) em áreas costeiras do Estado do Pará. Foram amostrados dois pontos, um no litoral (Praia do Crispim/Marapanim) e outro no estuário do rio Pará (Outeiro/Belém). As coletas foram feitas mensalmente em 2003 de abril a outubro em Outeiro e nos meses de junho, julho, setembro e outubro em Marapanim. Foram utilizados os métodos de isca humana e armadilha tipo CDC. Foram coletados 4003 exemplares de maruins distribuídos em 18 espécies de Culicoides Latreille e uma de Leptoconops Skuse, sendo L. brasiliensis (Lutz) a única espécie comum aos dois pontos de coleta. As duas espécies mais abundantes coletadas em Outeiro foram $C$. batesi Wirth \& Blanton e C. denisae Clastrier, enquanto em Marapanim foram C. maruim Lutz e C. phlebotomus (Williston).

Palavras-chave: Insecta, Diptera, Ceratopogonidae, Amazônia, zona costeira

\section{Introdução}

No Brasil os diptera hematófagos da família Ceratopogonidae são conhecidos vulgarmente como maruins, mosquito-pólvora e mosquitinho de mangue. Estes pertencem aos gêneros Culicoides Latreille, Forcipomyia Meigen subgênero Lasiohelea Kieffer e Leptoconops Skuse. O gênero Culicoides é o maior da família e também o mais distribuído. Segundo Mellor et al. (2000) mais de 1400 espécies de Culicoides são conhecidas em todo o mundo, das quais $96 \%$ atacam obrigatoriamente mamíferos (inclusive humanos) e aves. Estas espécies ocorrem em todas as grandes massas de terra, com exceção da Antártica e da Nova Zelândia, ocorrendo dos trópicos à tundra e do nível do mar até $4000 \mathrm{~m}$ de altitude.

Algumas espécies de Culicoides são vetores de protozoários e vermes filarídios, infectando aves, humanos e outros animais, mas é como vetores de viroses humanas e de animais que os Culicoides têm sua principal importância. Aqui no Brasil o vírus Oropouche causou algumas epidemias em humanos entre as quais cinco na região Amazônica, particu- 
larmente no Estado do Pará entre 1961 a 1972. Pinheiro et al. (1976) isolaram o vírus Oropouche em dois de quarenta lotes totalizando cerca de 1.500 Culicoides, dos quais $95 \%$ eram C. paraensis (Goeldi).

Acredita-se que o vírus da língua azul infecte todas as espécies conhecidas de ruminantes, mas as doenças severas usualmente ocorrem apenas em certas raças de ovelhas e algumas espécies de cervos. Este vírus é transmitido entre seus hospedeiros ruminantes quase exclusivamente pelo ataque de espécies de Culicoides vetores. Na América do Norte, $C$. variipennis (Coquillett) é o seu principal vetor e $C$. insignis Lutz e $C$. pusillus Lutz são os principais vetores no Sul da Florida, região Caribenha, na maior parte da América Central e em toda a América do Sul. (Mellor et al. 2000).

Apesar da importância epidemiológica dos ceratopogonídeos hematófagos, estes foram poucos estudados na Região amazônica, sendo os principais trabalhos realizados: Goeldi (1905) descreveu a espécie C. paraensis; Wirth \& Blanton (1973) estudaram o material coletado por Thomas H. G. Aitken com isca humana no nível do solo e em plataformas a 20 e $35 \mathrm{~m}$ na floresta APEG (Área de Pesquisas Ecológicas do Rio Guamá), Belém, e que resultou em quinze espécies novas para a ciência; Castellón (1990) coletou Culicoides com isca humana em três ambientes diferentes na Reserva Ducke, Amazonas, Brasil, sendo coletadas seis espécies; Castellón et al. (1990) trabalhando em três áreas da Amazônia brasileira, capturaram 22 espécies de Culicoides, das quais quatro não tinham sido encontradas ainda na Região amazônica; Castellón \& Ferreira (1991) coletaram as espécies $C$. todatangae Wirth \& Blanton, C. foxi Ortiz, C. fusipalpis Wirth \& Blanton e C. pseudodiabolicus Fox com isca humana em coletas noturnas na Reserva Ducke, Amazonas, Brasil; e Felippe-Bauer et al. (2000) descreveram espécie C. kampa proveniente de material coletado com armadilha luminosa no Estado do Acre.

Observa-se que os poucos trabalhos realizados com a entomofauna de maruins na Região amazônica foram principalmente em áreas de terra firme, deixando a vasta área costeira sem estudos, apesar de conter grandes populações destes insetos. O objetivo deste trabalho é levantar as espécies de ceratopogonídeos hematófagos em áreas costeiras do Estado do Pará, acrescentando informações úteis para o avanço das pesquisas e para controle nestes extensos ecossistemas na Amazônia brasileira. 


\section{Material e Métodos}

As coletas foram feitas em dois pontos da costa paraense, um na região nordeste (costa atlântica) e outro no estuário do Rio Pará. A região nordeste do estado do Pará apresenta grande diversidade de ambientes, destacando-se exuberantes manguezais, dunas, belas praias e restingas, sendo este último constituído por uma variedade de tipos vegetais. $\mathrm{Na}$ zona litorânea o ponto amostrado foi a Praia do Crispim, localizada no Município de Marapanim (Fig. 1).

O rio Pará não é propriamente um rio e sim um conjunto hidrográfico sem nascente própria, cujos seus principais formadores são os rios Araguaia, Tocantins, Capim, Acará, Moju, Guamá, Anapu, Jacundá, Pacajás e Araticum, cujas águas nele desembocam, dando origem a uma sucessão de baías e enseadas que se estendem ao longo de toda a costa sul da ilha de Marajó e o continente, desde a baía das bocas até ao mar, com mais de 300 quilômetros de extensão e cerca de 20 quilômetros de largura média (Lima et al., 2001). A influência do rio Amazonas no rio Pará é forte com suas águas vindas pelo furo de Breves. O grau de salinidade do rio Pará só se eleva próximo a sua foz, especialmente nos Municípios de Vigia, São Caetano de Odivelas, Salvaterra e Soure, sendo, portanto, nesses municípios que se observam extensos manguezais. Nesta zona estuarina o ponto amostrado foi Outeiro, mais precisamente a Praia da Brasília as margens do estuário do rio Pará, com forte influência das marés (Fig. 1).

As coletas foram feitas mensalmente em 2003 de abril a outubro em Outeiro e nos meses de junho, julho, setembro e outubro em Marapanim.

Foram utilizados dois métodos de coleta: isca humana e armadiIhas tipo CDC.

As coletas com isca humana foram feitas em dois períodos: um das 05:00 as 11:00h e o outro das 16:00h as 21:30h. Estes intervalos foram definidos baseados na literatura, na qual observa-se que a maioria das espécies de Ceratopogonidae (e em especial Culicoides) é crepuscular e também em observações anteriores feitas em áreas costeiras da Amazônia que evidenciaram que no intervalo de 10:00-17:00h, geralmente os maruins não estão atacando. Portanto, acredita-se que estes intervalos foram eficientes para cobrir a maioria das espécies que ocorreram nos locais de coleta durante o estudo.

Nas coletas com isca humana durante intervalos de 30 minutos, os maruins que procuraram os coletores foram capturados com auxílio 


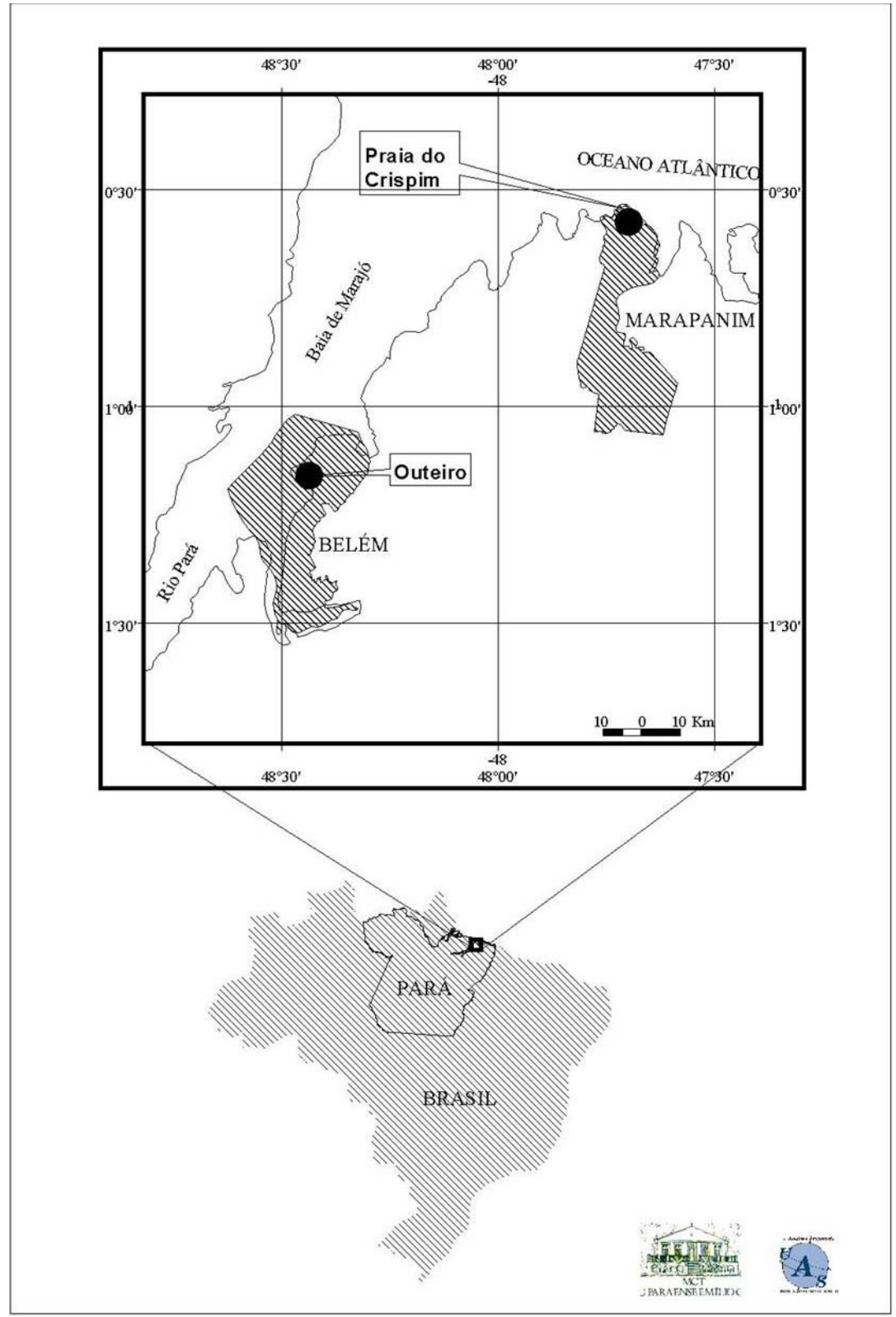

Figura 1: Pontos da costa do estado do Pará onde as coletas foram feitas: Praia do Crispim município de Marapanim costa atlântica e Outeiro município de Belém foz do rio Pará. 
de tubos de plástico tipo "ependorffs" (de 1,5 e 2,0 ml) contendo pedaço de papel toalha umedecido com acetato de etila. Esta técnica de usar "ependorffs" na captura de Ceratopogonidae hematófagos foi usada pela primeira vez. Observou-se que em ambientes que apresentam pequenas populações a técnica foi bastante eficiente, pois todos os exemplares que atacaram a isca foram facilmente coletados e já ficaram bem acondicionados. Porém, em ambientes com grandes populações não é possível coletar todos os exemplares que atacam a isca nos horários de pico.

Cada tubinho, contendo a amostra do intervalo de 30 minutos de coleta, foi devidamente etiquetado com o nome do coletor e horário de coleta e colocados dentro de sacos plásticos devidamente etiquetados por período de coleta. Os insetos coletados por este método permaneceram no interior dos ependorffs até que em laboratório fossem estudados.

Foram utilizadas também armadilhas luminosas do tipo CDC (Sudia \& Chamberlaim 1962), as quais foram instaladas nos locais de coleta as 18:00h e retiradas na manhã do dia seguinte as 7:00h. Os insetos coletados com este método foram mortos com acetato de etila e conduzidos até o laboratório no interior dos sacos coletores das armadilhas.

Alguns exemplares de cada amostra foram montados em lâminas de acordo com o método fenol-bálsamo de Wirth \& Marston (1968) para a identificação das espécies. Um maior número de exemplares foi guardado a seco em tubos de ensaio pequenos, devidamente etiquetados e identificados, os quais foram colocados dentro de frascos plásticos de rosca com naftalina. Todo esse material foi incorporado a Coleção de Invertebrados do Museu Paraense Emílio Goeldi.

Efetuou-se estimativa de riqueza de espécies em Outeiro e em Marapanim baseada nas coletas com isca humana. Utilizando para isto 0 programa Estimates 6.0 (Colwell 2000).

\section{Resultados e Discussão}

Durante este trabalho foram coletados 4003 exemplares de maruins distribuídos em 18 espécies de Culicoides e uma de Leptoconops. A tabela I apresenta a relação de espécies coletadas em Outeiro/Belém e na Praia do Crispim/Marapanim, no período de abril a outubro de 2003, através dos métodos de isca humana e armadilha tipo CDC. 
Tabela I

Número de exemplares por espécies de maruins capturados na zona costeira estuarina (Distrito de Outeiro, Belém) e no litoral atlântico (Praia do Crispim, Marapanim), no período de abril a outubro de 2003.

\begin{tabular}{|c|c|c|c|c|}
\hline \multirow[b]{2}{*}{ Espécies } & \multicolumn{2}{|c|}{ Locais de Coleta } & \multicolumn{2}{|c|}{ Métodos de Coleta } \\
\hline & Outeiro & $\begin{array}{l}\text { Praia do } \\
\text { Crispim }\end{array}$ & $\begin{array}{c}\text { Isca } \\
\text { Humana }\end{array}$ & CDC \\
\hline Culicoides acodylus Lutz & 03 & - & 03 & - \\
\hline C. betesi Wirth \& Blanton & 241 & - & 240 & 01 \\
\hline C. bimaculatus Floch \& Abonnenc & 07 & - & 06 & 01 \\
\hline C. cructier Clastrier & 42 & - & 42 & - \\
\hline C. doviesi Wirth \& Blanton & 04 & - & 04 & - \\
\hline C. debilipaipis Lutt & - & 07 & 07 & - \\
\hline C. cenisae Clastrier & 226 & - & 225 & 01 \\
\hline C. fox Ortiz & 01 & - & 01 & - \\
\hline C. furens (Poey) & - & 214 & 01 & 213 \\
\hline C. guyanensis Floch \& Abonnenc & - & 37 & 27 & 10 \\
\hline C. insignis Lutz & 18 & - & 17 & 01 \\
\hline C. yiartei Fox & 19 & - & 06 & 13 \\
\hline c. maruimLutz & - & 2434 & 678 & 1756 \\
\hline C. paraensis (Goeldi) & 12 & - & 12 & - \\
\hline C. paragnacioi Spinelli & 01 & - & 01 & - \\
\hline C. paramaruimWirth \& Blanton & 30 & - & 30 & - \\
\hline C. phebotomus (Williston) & - & 685 & 73 & 612 \\
\hline C. todatangaeWirth \& Blanton & 02 & - & 02 & - \\
\hline Leptoconops brasiliensis (Lut) & 01 & 19 & 20 & - \\
\hline Total & 607 & 3396 & 1395 & 2608 \\
\hline
\end{tabular}


Observa-se na tabela I que Leptoconops brasiliensis (Lutz) foi à única espécie comum a zona costeira estuarina e ao litoral atlântico, sendo, portanto, as espécies de Culicoides capturadas nos respectivos ambientes completamente diferentes e observa-se também que todas as espécies foram coletadas com isca humana. As duas espécies mais abundantes coletadas em Outeiro foram $C$. batesi e $C$. denisae, as quais foram coletadas principalmente com isca humana. Na Praia do Crispim, as duas espécies mais abundantes foram $C$. maruim e $C$. phlebotomus, coletadas mais freqüentemente com armadilhas $C D C$, sendo, portanto, diferente a eficiência das armadilhas nos dois ambientes amostrados (Outeiro/ estuário; Praia do Crispim/litoral). No estuário foram coletadas mais espécies, um total de 13 espécies de Culicoides e destas somente cinco $(38,46 \%)$ também foram coletadas com CDC; no litoral cinco espécies de Culicoides foram coletadas, das quais quatro (80\%) foram coletadas também com CDC. Segundo Mellor et al. (2000) as armadiIhas luminosas são aparentemente menos eficientes quando fontes alternativas de luz estão presentes e pode ter a eficiência reduzida nas noites de lua. Acredita-se que em Outeiro o pequeno número de exemplares coletados com armadilhas tipo CDC, mesmo da espécie mais abundante $(C$. batesi) que ocorreu em todos os horários do período noturno em que as coletas com isca humana foram realizadas, tenha sido devido à influência de luz artificial, pois em Outeiro havia bastante interferência de lâmpadas acesas, enquanto que em Marapanim havia apenas luz natural e das fontes luminosas das armadilhas. O tamanho das populações também deve ter influenciado.

Quanto à riqueza de espécies e abundância de indivíduos observase que os dados deste trabalho seguiram o que se esperava na comparação do litoral com outros ambientes, pois em Marapanim o número de espécies de Culicoides foi pequeno (cinco), porém mais abundantes (3377 exemplares), enquanto em Outeiro o número de espécies foi maior (13) com abundâncias menores (606 exemplares). Apesar do método de isca humana ter sido mais eficiente neste estudo para coletar espécies de importância médica, sugere-se que os dois métodos (isca humana e armadilha CDC) sejam utilizados na amostragem de Ceratopogonidae, pois os machos das espécies hematófagas e as espécies predadoras são coletados com armadilhas. A tabela II indica o número de machos e fêmeas por espécies de maruins coletados em Outeiro e na Praia do Crispim com CDC. 


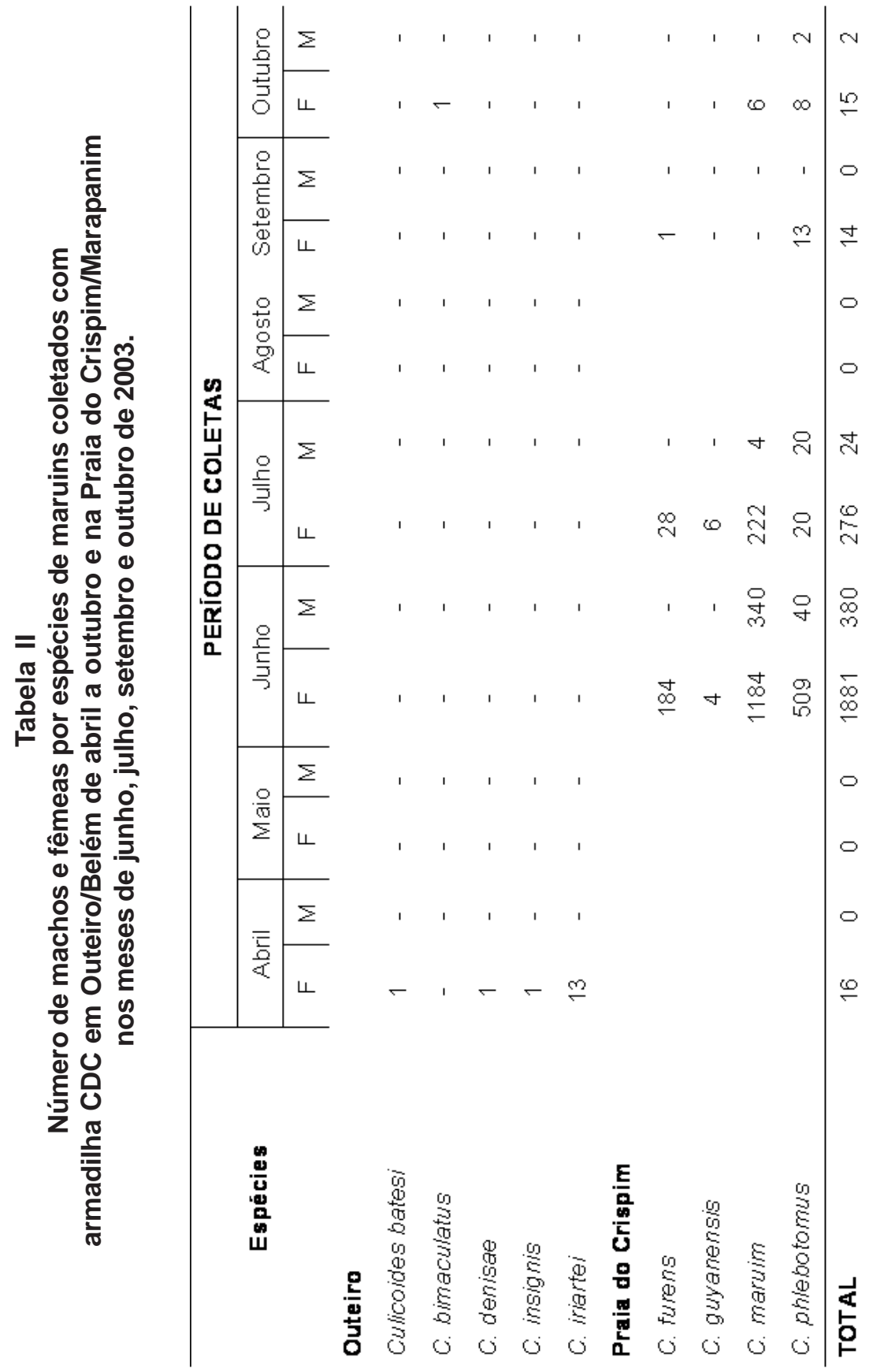


De acordo com Borkent \& Spinelli (2000) C. crucifer ocorre apenas em Trinidad, Guiana e Guiana Francesa e C. daviesi no Peru e na Guiana. C. crucifer foi descrita por Clastrier (1968) baseado em exemplares machos e fêmeas provenientes da Guiana Francesa e C. daviesi foi descrita por Wirth \& Blanton (1968) baseados em exemplares fêmeas provenientes da Guiana. Este trabalho amplia a distribuição destas espécies para o norte do Brasil.

Ampliam-se também as distribuições de $C$. denisae, C. phlebotomus e Leptoconops brasiliensis no Brasil, agora com registros no Estado do Pará, pois segundo Borkent \& Spinelli (2000) C. denisae ocorre na Guiana Francesa e no Brasil (Estado do Amazonas); C. phlebotomus em áreas costeiras do México (Sinaloa) ao Equador e da Jamaica ao Brasil (com registro nos Estados do Maranhão, Ceará, Pernambuco e Goiás) e L. brasiliensis ocorre no Brasil (Estado do Amazonas) e Uruguai. Um ajuste quanto à distribuição de $L$. brasiliensis é apresentado aqui, pois, de acordo com Lutz (1913), o autor desta espécie, o maior número de exemplares fêmeas por ele analisado é procedente da parte inferior do rio Tocantins, sendo, portanto, L. brasiliensis proveniente do Estado do Pará e não do Amazonas.

A ausência de registros destas espécies no Estado do Pará se deve à falta de levantamentos da entomofauna de maruins na Amazônia, principalmente em áreas costeiras, sendo este o primeiro com este propósito.

Observa-se na tabela Il que em Outeiro não foram coletados exemplares machos das espécies de Culicoides com CDC, enquanto que em Marapanim coletou-se machos apenas das duas espécies mais abundantes (C. maruim e $C$. phlebotomus) e que os exemplares de $C$. furens foram coletados quase exclusivamente com CDC, exceto um exemplar que foi coletado com isca humana. Segundo Aitken et al. (1975) a maioria das coletas de $C$. furens provenientes de Trinidad e Tobago que cobre 0 período de 1955 a 1970 são de armadilhas luminosas. Talvez, C. furens seja mais abundante em coletas com armadilhas luminosas devido seu horário noturno de pico de atividade.

Realizou-se estimativa da riqueza de espécies separadamente para Outeiro e Marapanim baseada nas coletas realizadas com isca humana (Fig. 2).

Em Outeiro a estimativa de riqueza de espécies variou de 15 a 19 espécies para um número observado de 14 espécies em 164 amostras. Bootstrap estimou o menor número de espécies (15), enquanto, Jack 2 estimou o maior número de espécies (19). 


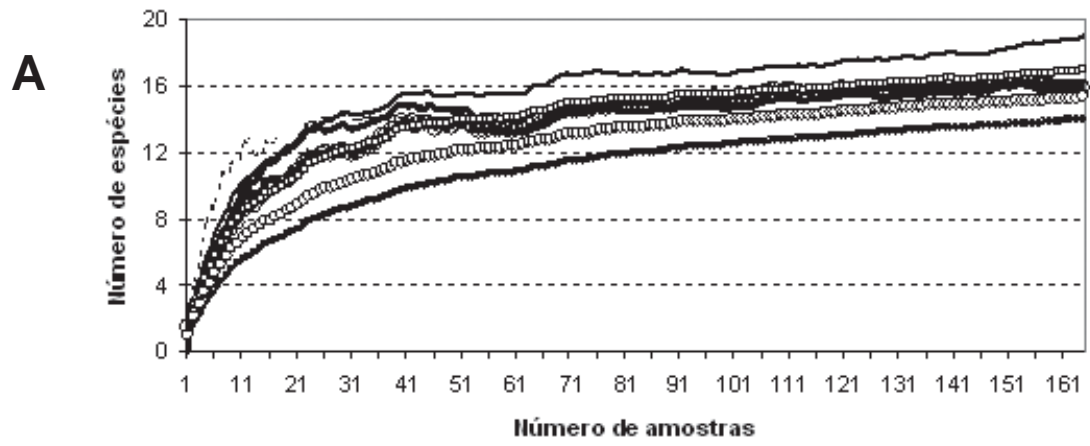

B

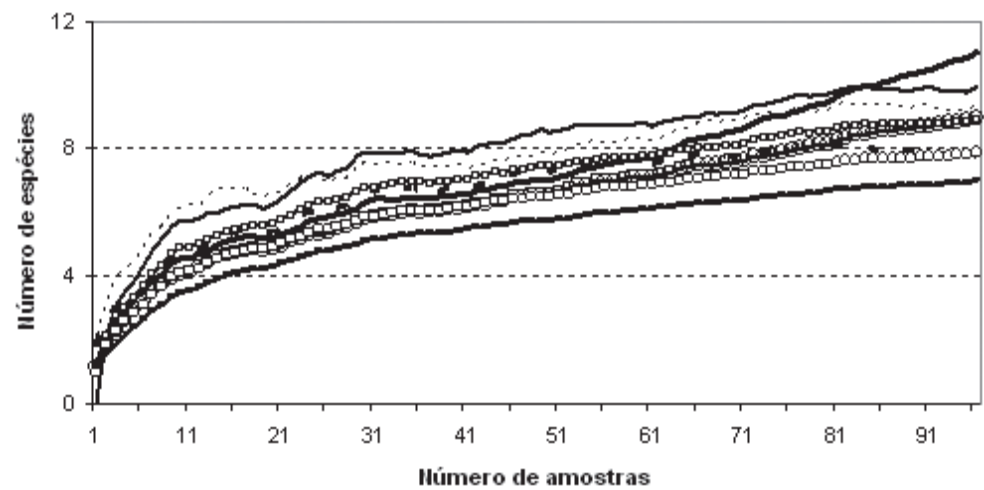

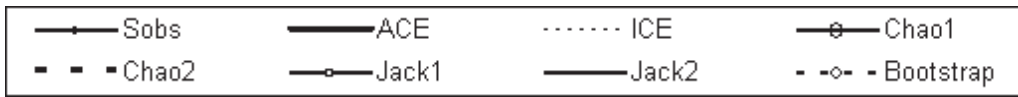

Figura 2: Valores obtidos para estimar a riqueza de espécies das amostras dos estimadores ACE, ICE, Chao1, Chao2, Jack1, Jack2 e Bootstrap (Colwell, 2000). Obtidas em: A - Outeiro (Belém); B - Praia do Crispim (Marapanim).

Na Praia do Crispim em Marapanim a estimativa de riqueza de espécies variou de 8 a 11 espécies para um número observado de 7 espécies em 96 amostras. Chao 2 estimou o menor número de espécies (8), enquanto, ACE estimou o maior número de espécies (11). 
Observa-se, tanto em Outeiro como em Marapanim, que as curvas geradas pelos estimadores (ACE, ICE, Chao 1, Chao 2, Jack 1, Jack 2 e Bootstrap) caracterizam a necessidade de mais amostras, as quais deverão cobrir pelo menos um período anual. Os meses do período chuvoso não foram devidamente amostrados nas duas localidades estudadas, pois as coletas foram iniciadas em Outeiro no mês de abril (final do período chuvoso) e em Marapanim em junho (início do período seco). Entretanto, observou-se que em Outeiro o maior número de espécies foi coletado no mês de abril (10), das quais três foram coletadas apenas neste mês; em Marapanim o maior número de espécies foi coletado no mês de junho (seis), das quais quatro foram coletadas com isca humana apenas neste mês e destas $C$. debilipalpis e $L$. brasiliensis ocorreram somente em junho. Portanto, para melhor caracterizar a entomofauna de maruins em áreas costeiras do Estado do Pará, nos respectivos ambientes do litoral e estuário do rio Pará é necessário um estudo mais abrangente, com mais pontos amostrados e que cubram períodos anuais.

\section{Agradecimentos}

À Coordenação de Aperfeiçoamento de Pessoal de Nível Superior (CAPES) pelo apoio com a bolsa durante o curso de mestrado. Ao Museu Paraense Emílio Goeldi (MPEG) e ao Programa de Pós-graduação em Zoologia da UFPA/MPEG que viabilizaram o desenvolvimento deste trabalho. À pesquisadora Maria Luiza Felippe-Bauer do Instituto Oswaldo Cruz, Rio de Janeiro, RJ, pelas importantes informações sobre montagem, conservação e identificação de Ceratopogonidae. Ao pesquisador Gustavo Espinelli da Divisão de Entomologia do Museu de La Plata, Argentina, pelo apoio na literatura relativa a taxonomia. Aos técnicos Artur Lobão, Domingos Dalcides e Luzanira Seleiro da Entomologia do Museu Paraense Emílio Goeldi e a amiga Ivaneide Furtado pela ajuda durante as coletas de ceratopogonídeos. À funcionária Arlete Silva de Almeida do Museu Paraense Emílio Goeldi pela composição do mapa das áreas de estudo. 


\section{Referências Bibliográficas}

Aitken, T.H.G.; Wirth, W.W.; Williams, R.W.; Davies, J.B. \& Tikasingh, E.S., 1975. A review of the bloodsucking midges of Trinidad and Tobago, West Indies (Diptera: Ceratopogonidae). J. Entomol. 44:101-144.

Borkent, A. \& Spinelli, G.R., 2000. Catalog of the New World Biting Midges South of the United States of America (Diptera: Ceratopogonidae). Contrib. Entomol. Internatl. 4:1-107.

Castellón, E.G., 1990. Culicoides (Diptera: Ceratopogonidae) na Amazônia brasileira. II. Espécies coletadas na Reserva Florestal Duck, aspectos ecológicos e distribuição geográfica. Acta Amazonica 20:83-93.

Castellón, E.G. \& Ferreira, R.M., 1991. Culicoides Latreille (Diptera: Ceratopogonidae) da Amazônia. III. Resultados de coletas noturnas, na Reserva Florestal Ducke, Estado do Amazonas, Brasil. Bol. Mus. Para. Emílio Goeldi, sér. Zool. 7:117-123.

Castellón, E.G.; Ferreira, R.L.M. \& DASilva, M.N.T., 1990. Culicoides (Diptera: Ceratopogonidae) na Amazônia brasileira. I. Coletas na usina hidroelétrica (UHE) de Balbina, Usina Hidroelétrica (UHE) Cachoeira Porteira e Cachoeira dos Espelhos (Rio Xingu). Acta Amazonica 20: 77-81.

Clastrier, J., 1968. Deux Ceratopogonides nouveaux de la Guyane Francaise. Arch. Inst. Pasteur Guyane Franc. L'Inini 21:85-92.

Colwell, R. K., 2000. Estimates. Statistical estimation of species richness and shared species from samples. Disponível em: http:// viceroy.eeb.uconn.edu//Estimates. Acesso em 27 de janeiro de 2004.

Felippe-Bauer, M.L.; Veras, R.S.; Castellón, E.G \& Moreira, N.A., 2000. A New Culicoides from The Amazonian Region, Brazil (Diptera: Ceratopogonidae). Mem. Inst. Oswaldo Cruz 95:35-37.

Goeldi, E., 1905. Os mosquitos no Pará. Reunião de quatro trabalhos sobre os mosquitos indígenas, principalmente as espécies que molestam o homem. Mem. Mus. Paraense Emilio Goeldi Hist. Nat. Ethnogr. 4:1-154. 
Lima, R.R.; Tourinho, M.M. \& Costa, J.P.C. da., 2001. Várzeas flúviomarinhas da Amazônia brasileira: características e possibilidades agropecuárias. FCAP, Serviço de Documentação e Informação, Belém, Pará, $342 \mathrm{p}$.

Lutz, A., 1913. Contribuição para o estudo das Ceratopogoninas hematofagas do Brazil. Mem. Inst. Oswaldo Cruz 5:45-73.

Mellor, P.S.; Boorman, J. \& Baylis, M. 2000. Culicoides biting midges: their role as arbovirus vectors. Ann. Rev. Entomol. 45:307-340.

Pinheiro, F.P.; Travassos da Rosa, A.P.A.; Travassos da Rosa, J.F. \& Bensabath, G., 1976. An outbreak of Oropouche virus disease in the vicinity of Santarem, Para, Brazil. Tropenmed Parasitol. 27:213-223.

Sudia, W.D. \& Chamberlain, R.W., 1962. Battery-operated light trap, an improved model. Mosq. News 22:126-129.

Wirth, W.W. \& Blanton, F.S., 1968. A new Culicoides from Guyana (Diptera: Ceratopogonidae). Fla. Entomol. 51:251-252.

Wirth, W.W. \& Blanton, F.S., 1973. A review of the maruins or biting midges of the genus Culicoides (Diptera: Ceratopogonidae) in the Amazon Basin. Amazoniana 4:405-470.

Wirth, W.W. \& Marston, N., 1968. A method for mounting small insects on microscope slides in Canada balsam. Ann. Entomol. Soc. Am. 61:783-784. 